\title{
Performance Analysis of Dynamic Threshold Estimation Techniques Based on the One-Tier Cognitive Radio Network
}

\author{
Aditi Gupta1 ${ }^{*}$, Adeiza James Onumanyi ${ }^{2}$ \\ ${ }^{1}$ Department of Electrical Engineering, Indian Institute of Technology, Jammu, India \\ ${ }^{2}$ Department of Telecommunication Engineering, Federal University of Technology, Minna, Nigeria \\ Email: *aditiguptaaug93@gmail.com
}

How to cite this paper: Gupta, A. and Onumanyi, A.J. (2019) Performance Analysis of Dynamic Threshold Estimation Techniques Based on the One-Tier Cognitive Radio Network. Journal of Computer and Communications, 7, 31-46. https://doi.org/10.4236/jcc.2019.72003

Received: December 30, 2018

Accepted: February 15, 2019

Published: February 18, 2019

Copyright ( 2019 by author(s) and Scientific Research Publishing Inc. This work is licensed under the Creative Commons Attribution International License (CC BY 4.0).

http://creativecommons.org/licenses/by/4.0/

\begin{abstract}
Cognitive Radios (CRs) use dynamic threshold estimation (DTE) techniques to better detect primary user signals under noise uncertainty regimes. However, DTE techniques have rarely been compared before, particularly under the one tier CR network (CRN) model, making it difficult to assess their comparative performance characteristics under this regime. Thus, in this paper, we have investigated the performance of some notable DTE methods under the one-tier CRN model. We used the auction game model in our investigation to compare fairly the spectrum efficiency performance of each technique. Our findings show that DTEs generally perform better than the fixed threshold method particularly under unpredictable noise uncertainty regimes. Our results show further that the channel utilization $(\mathrm{CU})$ rate of the fixed threshold method, popularly used by researchers, plummets by $50.26 \%$ for a $1 \mathrm{~dB}$ increase in the noise uncertainty level, while the $\mathrm{CU}$ rate of the DTE techniques interestingly increased by an average of $4 \%$. Our investigation will enable CR Engineers to better understand the performance characteristics of DTE techniques under the one-tier CRN model.
\end{abstract}

\section{Keywords}

Adaptive Threshold, Auction Game, Cognitive Radio, Energy Detection, One-Tier

\section{Introduction}

Recently, the need to improve user perceived quality of service (QoS) in newer wireless technologies has increased the demand for more radio spectrum. This demand is worrisome for many wireless industry players because of the obvious 
lack of more spectrums to allocate to new wireless technologies, which may eventually degrade wireless QoS in the end. In this regard, researchers have suggested the need to exploit existing but underutilized spectra, called whitespaces (or spectrum holes) [1] [2] towards improving QoS. To achieve this, many consider Cognitive Radio (CR) as the candidate technology to exploit these whitespaces in order to enhance user perceived QoS in new wireless technologies. CR detects the presence or absence of Primary User (PU) signals in a channel and either uses the free channel to communicate (if PU signals are absent) or vacates the channel quickly enough (if PU signals are present) to prevent interference [3] [4] [5].

There are many techniques for spectrum sensing (SS) in CR including the Cyclostationary, Matched Filter, Compressed sensing, and the Energy Detection (ED) methods. However, many authors widely designate the ED as the most viable method because it provides the lowest computational complexity and it is the simplest approach amongst all [6] [7] [8] [9] [10]. The ED does not require prior knowledge of the PU signal waveform, thus making it a suitable blind SS technique for CR [6]. Nevertheless, noise variations and dynamic spectra conditions in the communication channel often limit the ED's performance because of their dependence on static thresholds [11]. Consequently, recent studies have proposed the use of dynamic threshold estimation (DTE) methods in order to improve the ED's robustness to noise uncertainties.

In this paper, we have investigated the performance of some notable DTE techniques under the one tier CRN model including the Modified Otsu method, Forward Consecutive Mean Excision (FCME), Recursive One sided Hypothesis Testing (ROHT) and the First Order Statistical Technique (FOST). Since the one tier CRN refers to the basic CRN model wherein secondary users simply transmit opportunistically in a PU band, thus, we considered it worthwhile to commence our investigation of DTE techniques based on this basic model of a CRN. We used the auction game model in order to provide a fair chance to all available users in the CRN, which further guarantees improved spectrum efficiency. The auction game model allows the user with the highest utility metric to use the spectrum first and subsequent users then follow in respect of their utility values. The DTE techniques considered here typically require less than two parameters to be tuned making them quite simple and viable methods. Following our findings, we pose the contributions of this paper as follows:

1) We investigate for the first time the performance of DTE techniques using the auction game model for the one-tier CRN.

2) We provide statistical results that demonstrate the better performance of DTE techniques over the fixed threshold method.

3) Our results reveal particular conditions under which the different DTE techniques may best perform, thus enabling the CR Engineer to apply and develop better systems for CR applications.

We organise the rest of this paper as follows: Section 2 provides a brief review of related works while Section 3 describes the system model, and Section 4 de- 
scribes the different DTE techniques that we investigated. We present and discuss our findings in Section 5 and conclude in the Section 6.

\section{Relate Work}

Many studies have proposed a number of DTE methods and we discuss a few to motivate our research. Authors in [12] proposed the double threshold method. It has two pre-fixed threshold values used to detect PU signals. Essentially, the double threshold method detects a signal only when the signal's energy surpasses the two pre-fixed threshold values. However, the study in [12] only compared the double threshold method with the fixed threshold method and not with other adaptive threshold methods. This limits our understanding of its comparative performance characteristics. In [13], authors developed the adaptive double threshold method, which is an upgrade to the approach in [12]. In [13], authors compared their method only with the ordinary double threshold method and the adaptive spectrum sensing method under noise uncertainty, and not with other adaptive methods. Authors in [14] introduced the idea of hierarchal cooperative spectrum sensing. Similar to the double threshold methods discussed in [12] [13]. They proposed the concept of soft combination of the observed energy falling between the two thresholds [14]. Authors compared their approach only with the conventional double threshold and the n-Ratio detection method, which indicates the need for wider comparisons. Other DTEs are mentioned in [15]-[22], for example the two-stage detectors and the utilization of fuzzy logic for threshold selection. These studies were likewise limited in their depth of comparisons with other DTE methods.

Furthermore, in our study, we considered only simple and effective techniques, particularly those that have two or less parameters to optimize. A popular viable DTE technique with two tuneable parameters is the forward methods discussed in [23] [24]. Specifically, Lehtomaki et al., in [23] [24] used two different forward methods as DTEs by rearranging the measured sample sets to determine appropriate threshold values. They advanced their work in [25] [26] to develop the Forward Consecutive Mean Excision (FCME) method and other variants like the Median FCME (MED-FCME). Though highly effective, these methods were rarely compared with other viable DTE methods, thus limiting our knowledge of their comparative performance characteristics. Other DTE methods are discussed in [27] [28] [29] [30] [31] including the Recursive One sided Hypothesis Testing (ROHT) discussed in [32] [33] and the First Order Statistical Test (FOST) proposed in [34] and Modified Otsu's algorithm in [35]. Barnes et al., in [36] also proposed a similar method to the ROHT, which differs from the ROHT only by the use of the probability density function to determine the noise level. The ROHT was compared only with the Otsu method in [33], while Barnes et al. compared their method only with the ROHT. These limited comparisons further indicate the need for wider comparative analysis.

In [32] [33], Datla et al., used the Otsu algorithm as a DTE method for signal 
detection in CR. Onumanyi et al., modified Datla's approach in order to overcome limitations such as the cumbersome need for grey level computations and the poor performance in noise only conditions [35]. Onumanyi et al., compared his method with the original Otsu method and the ROHT. Some game theory based approaches have been proposed to optimize spectrum use for the one-tier CRN [37] [38]. In [39], a two tier CRN was developed that consists of one tier for the primary users and the two tiers for the secondary users comprising of real and non-real time users. In [40], authors used the auction game model to optimize the two-tier CRN overcoming the limitations of the approach in [39].

In our work, we introduced the auction model for the first time to investigate the performance of a wider range of notable DTE methods under the one-tier CRN model. To the best of our knowledge, and different from existing DTE studies, our investigation determines for the first time the comparative performance characteristics of a wider range of viable DTE methods particularly under the auction game model. Our study becomes quite instructive for CR Engineers because we use a wider range of metrics to compare these methods.

\section{System Model}

We considered the Energy Detection (ED) method as the basis for our investigation. The DTE methods we considered were deployed in the ED and their respective performances were investigated. We used the auction game model to optimize the transmission opportunities of users in the one tier CRN. We show in Figure 1 the overall system model including its three basic sections: the energy detector section, the automatic/dynamic threshold estimator block and the auction game model block based on the one tier CRN.

The input signals were processed by the ED block beginning with the band pass filter that determines the channel of interest. In the band pass filter, we fixed the band of the channel by setting the lower and upper input signal frequency of the ED. The ED then down-converts the band-passed signals to lower intermediate frequency bands and digitizes these signals in the analogue to digital converter (ADC) block. It computes the signal energy using the k-point Fast

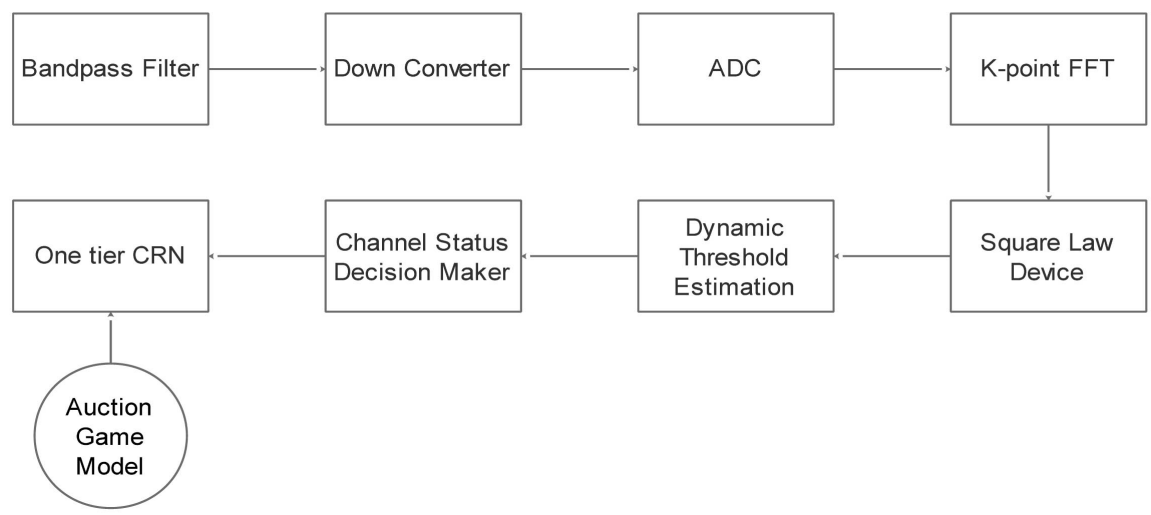

Figure 1. Energy detector model with automatic threshold estimation for optimized one tier Cognitive Radio Network (CRN). 
Fourier Transform (FFT) and squares these values before integrating the signal samples.

The ED then compares the energy of each signal sample to a threshold value in the automatic threshold estimator block to detect the presence or the absence of PU signals in the channel. The output of the decision is passed through the auction game model, which we shall describe in Section 4. Nevertheless, in the theoretical sense, let $\gamma(n)$ be the received signal generally expressed as:

$$
\gamma(n)=u(n)+w(n)
$$

where, $u(n)$ is the transmitted primary signals, $w(n)$ represents Additive White Gaussian Noise (AWGN). The decision metric for the ED model is mathematically expressed as:

$$
\chi=\sum_{n=0}^{\mathcal{N}}|\gamma(n)|^{2}
$$

where, $\mathcal{N}$ is the observation interval. In order to determine whether the channel is vacant or not, the signal energy measured in (2) is compared to the threshold value $\lambda$. The absence or presence of PU signals in a channel is denoted as $\mathcal{H}_{0}$ and $\mathcal{H}_{1}$, respectively.

$$
\begin{gathered}
\gamma(n)=w(n): \mathcal{H}_{0} \\
\gamma(n)=u(n)+w(n): \mathcal{H}_{1}
\end{gathered}
$$

where, $\mathcal{H}_{0}$ denotes the noise only spectrum (PU signal is absent) and $\mathcal{H}_{1}$ represents the signal plus noise condition or the presence of $\mathrm{PU}$ signal in the channel.

We used the probability of detection $\mathcal{P}_{\mathcal{D}}$ and the probability of false alarm $\mathcal{P}_{\mathcal{F A}}$ to analyse the performance of the detector based on the different DTE methods. Both the $\mathcal{P}_{\mathcal{D}}$ and the $\mathcal{P}_{\mathcal{F A}}$ are mathematically described as:

$$
\begin{gathered}
\mathcal{P}_{\mathcal{D}}=\mathcal{P}_{r}\left(\mathcal{X} \geq \lambda \mid \mathcal{H}_{1}\right) \\
\mathcal{P}_{\mathcal{F A}}=\mathcal{P}_{r}\left(\mathcal{X}>\lambda \mid \mathcal{H}_{0}\right)
\end{gathered}
$$

The threshold value $\lambda$ needs to be carefully selected in order to achieve a balance between the $\mathcal{P}_{\mathcal{D}}$ and $\mathcal{P}_{\mathcal{F A}}$ rate. Since the threshold is highly susceptible to changes in the noise level, which may limit the ED's performance, thus we investigated the use of DTE methods. We investigated four different DTE techniques namely: the Modified Otsu algorithm, ROHT, FOST and the FCME techniques, which we shall explain further in Section 4.

\section{Dynamic Threshold Techniques and the Auction Game Model}

\subsection{Dynamic Threshold Techniques}

There are various dynamic thresholding techniques and we investigated the following methods.

\subsubsection{Recursive One-Sided Hypothesis Testing (ROHT)}

The ROHT algorithm recursively estimates an appropriate threshold value for 
signal detection [32] [33]. We considered the ROHT algorithm in our research for its efficiency, effectiveness, and simple design [32] [33]. Thus, we describe its process in this section based on the flowchart of Figure 2 . The threshold function used in the ROHT algorithm is $\lambda_{i+1}=\mu_{i}+Z \cdot \sigma_{i}$ where $Z$ is the coefficient of the standard deviation, $\lambda_{i+1}$ is the threshold value that we seek to estimate, $\mu_{i}$ is the mean of $\mathcal{N}$ measured samples, and $\sigma_{i}$ is the standard deviation of the measured sample set.

According to [32], the ROHT algorithm initializes the set of measured signal samples as an empty set, $S_{0}$. It then computes an initial decision threshold using $\lambda_{i+1}=\mu_{i}+Z \cdot \sigma_{i}$. The ROHT algorithm uses $\lambda_{i+1}$ to classify the input signal into two exclusive sets, each containing signal samples, $S_{i+1}$, and the noise samples, $N_{i+1}$, respectively. The signal set, $S_{i+1}$ contains samples with values greater than $\lambda_{i+1}$, while samples with values less than $\lambda_{i+1}$ are considered as noise samples.

The ROHT algorithm expunges the signal samples from the input signal set and keeps only the noise samples, $N_{i+1}$. It iterates until a stopping criterion, $\beta$ is met, which occurs when the difference between two consecutive standard deviation values is less than a specified positive value, $\beta$. The algorithm considers the estimated threshold value at the stopping criterion as the final threshold value for signal detection.

\subsubsection{First Order Statistical Technique (FOST)}

The FOST shares a degree of similarity to the ROHT algorithm. The FOST uses the same threshold function as the ROHT algorithm but excludes its recursive mechanism [36]. Thus, the ROHT algorithm reduces to the FOST when it is iterated only once. Consequently, the FOST is a faster version of the ROHT algorithm. We considered the FOST in order to analyse how its speed may affect threshold performance in the one-tier CRN model.

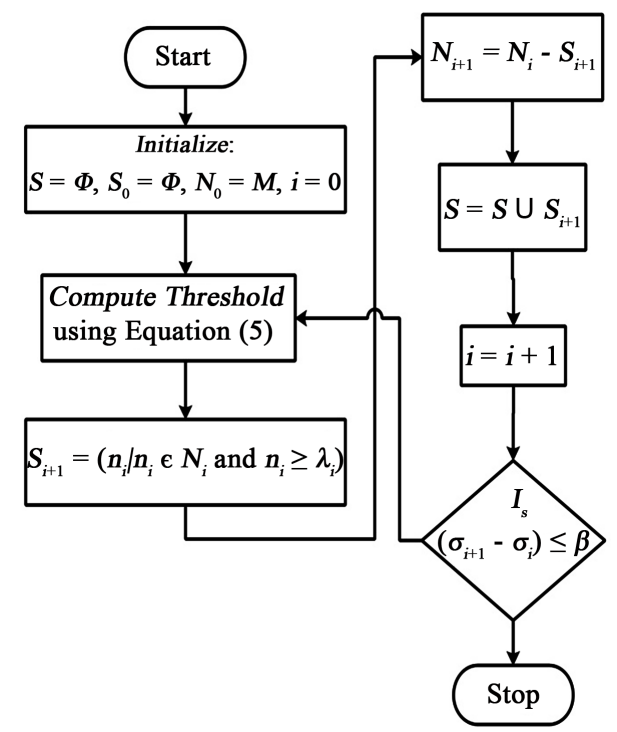

Figure 2. The ROHT algorithm. 


\subsubsection{Forward Consecutive Mean Excision (FCME)}

The FCME algorithm is an efficient, effective and simple algorithm and it has been used extensively in different CR applications. We depict the FCME algorithm in Figure 3. According to [23], the FCME algorithm accepts the estimated energy samples $\gamma(n)$ as input. Usually, the $T_{c m e}$ and $Q$ parameters are calculated a priori (manually) at the start of the FCME process. The algorithm rearranges the samples $\gamma(n)$ in an ascending order and processes the first $Q$ samples in $\gamma(n)$, i.e. for $n=1,2, \cdots, Q$. The mean of the first $Q$ samples is obtained as $N e$ and an initial threshold value is computed as $\lambda_{i}=T_{\text {cme }} \cdot N e$. The algorithm iterates until an outlier is found, which terminates the algorithm and converges to a final $\lambda$ value.

\subsubsection{Modified Otsu's Algorithm}

The Modified Otsu's algorithm (MOA) is an adaptive and autonomous threshold estimation technique proposed in [35] for CR application. Essentially, the MOA achieves an averagely lower false alarm rate as compared to the original version proposed in [32]. It is independent of the bandwidth size and has a total complexity of $\mathrm{O}(\mathrm{N})$ where $\mathrm{N}$ is the total sample size. The MOA provides complete and automatic blind spectrum sensing for CR applications. For these reasons, we considered the MOA in our investigation. However, for further details of its function, we kindly refer our readers to [35].

\subsection{Auction Game Model}

We describe the auction game model used in the one tier CRN to ensure that all users get a fair chance to use the spectrum. The auction game model considers all users as cognitive radio users and allows them to sense the spectrum for possible transmission [40]. After sensing, users make a collaborative decision on the

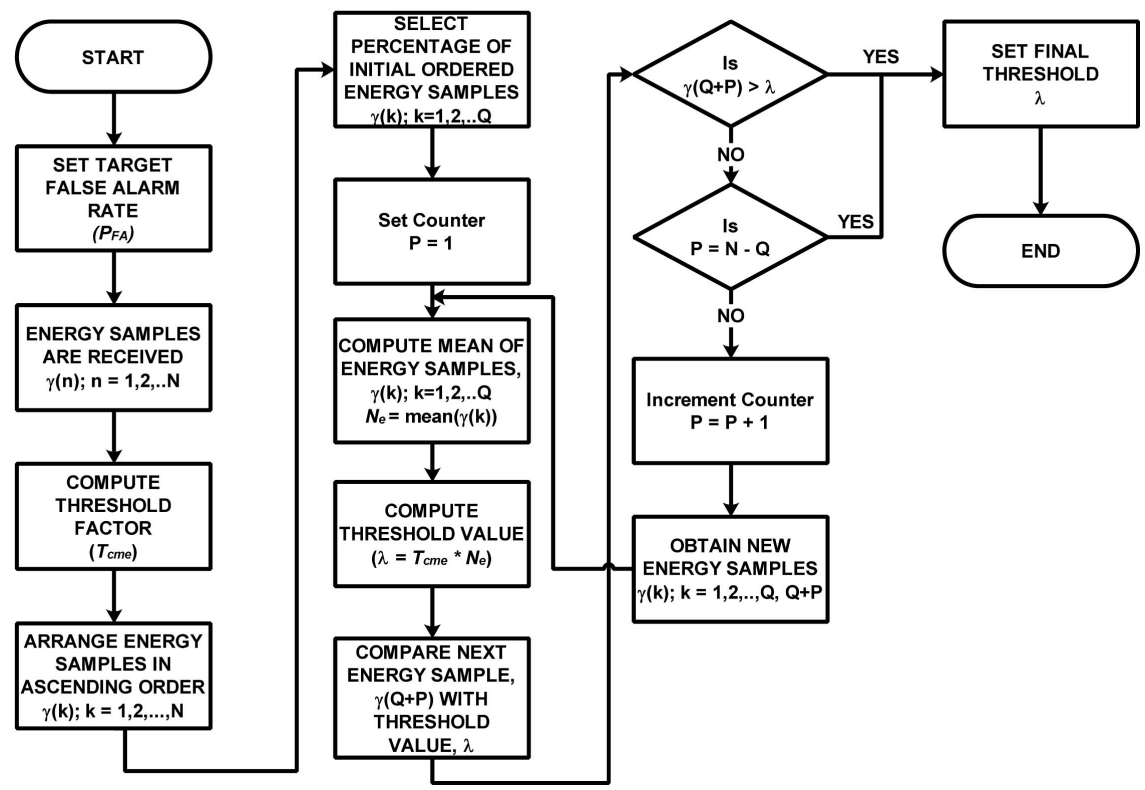

Figure 3. The FCME algorithm. 
availability of channels. A channel availability vector is then broadcasted by the base station to all users. A reward payoff matrix and a price matrix are updated and users are allotted the available channels depending upon the maximum utility metric. We describe each metric in the auction game model as follows.

\subsubsection{Detection Metric}

Detection metrics used are the probability of detection $\mathcal{P}_{\mathcal{D}}$ and the probability of false alarm $\mathcal{P}_{\mathcal{F A}}$. The $\mathcal{P}_{\mathcal{D}}$ denotes the probability of detecting the primary user by a secondary user in a channel while the $\mathcal{P}_{\mathcal{F A}}$ denotes the probability of wrongly alarming the presence of the primary user in a channel when it is not present. Mathematically, $\mathcal{P}_{\mathcal{D}}$ and $\mathcal{P}_{\mathcal{F A}}$ for each channel and for each user is expressed as:

$$
\begin{aligned}
& \mathcal{P}_{\mathcal{D}}=\frac{1}{2} \operatorname{erfc}\left(\frac{\lambda-u}{\sqrt{2 w}}\right) \\
& \mathcal{P}_{\mathcal{F A}}=\frac{1}{2} \operatorname{erfc}\left(\frac{\lambda}{\sqrt{2 w}}\right)
\end{aligned}
$$

where $\lambda$ is the threshold value obtained using the DTE, while $\mu$ and $\omega$ are the signal and noise values, respectively.

\subsubsection{Decision Matrix}

The decision matrix is used to decide whether the primary users are present or not. The decision matrix $D_{c_{i}}^{a_{j}}$ represents the decision taken by each cognitive user $c_{i}$ per channel $a_{j}$. We obtain $D_{c_{i}}^{a_{j}}$ as:

$$
\begin{gathered}
D_{c_{i}}^{a_{j}}=\left\{\begin{array}{l}
1: H_{1}, \mathcal{X} \geq \lambda \\
0: H_{0}, \mathcal{X}<\lambda
\end{array}\right. \\
\xi=\sum_{i=1}^{K} D_{c_{i}}^{a_{j}}
\end{gathered}
$$

where $K$ is the total number of cognitive radio users in the system that want to transmit, and $\xi$ represents the overall sum of the decision of each user per channel. The decision matrix gives the result in the form of " 1 " or " 0 " for the presence or absence of the PU signal.

\subsubsection{Channel Availability Vector}

The channel availability vector $A$ comprises of the available channels for the transmission of the secondary users. This vector depends on the decision matrix as well as on the " $n$-out of-k" voting rule [35]. The channel availability vector is expressed as:

$$
A=A \bigcup\left\{a_{j}\right\} \text { if } \xi<n
$$

We considered $n=0.5$ in our investigation, that is, the channel is considered free if $50 \%$ of the entire users in the CRN vote that the channel is free.

\subsubsection{Reward Payoff Matrix}

The reward payoff matrix $\pi$ is defined as the payoff received by a user for 
transmitting over a particular channel $a_{j}$. It is expressed as:

$$
\pi=\left\{\alpha_{c_{i}}^{a_{j}}\right\}
$$

where

$$
\alpha_{c_{i}}^{a_{j}}=\frac{B}{N_{c}} \log _{2}\left(1+S N R_{c_{i}, a_{j}}\right)
$$

Here $B$ is the total bandwidth while $S N R$ is the signal to noise ratio for each user transmitting on a channel and $N_{c}$ is the total number of channels.

\subsubsection{Price Vector}

The price vector $\theta$ denotes the price that a user pays to obtain a particular channel for transmission and it is obtained as:

$$
\theta=\frac{\phi}{G}
$$

where $G$ is the gain of each user for each channel and the $\phi$ represents the urgency of transmission, which depends on the number of packets a user needs to transmit. The value of $\phi$ can be randomly chosen per user between 0.1 and 0.9 where 0.1 indicates the highest priority and 0.9 indicates the lowest priority of the user for the transmission.

\subsubsection{Utility Function}

The auction game chooses the user and the channel that pays the minimum price and the maximum reward. Thus, the aim is to maximize the utility function $\Omega$ expressed as:

$$
\Omega=(\pi-\theta) \times \mathcal{P}_{\mathcal{D}}
$$

Two additional parameters are taken into the consideration namely the Channel utilization (CU) and the User Service Rate (USR). The CU indicates in percentages how many channels are being utilized after applying the auction game model and the USR indicates how much the auction model serves the users. Both of these parameters are very important as far as the transmission is taken into the consideration because these helps us to know which among the many dynamic thresholding techniques is better performing considering the one-tier cognitive radio network.

\section{Results and Discussion}

In this section, we present and discuss the results obtained from simulations conducted using the different DTE techniques under the one-tier CRN based on the auction game model. We compared these DTE techniques with the fixed threshold method to show the performance limit of the fixed method under noise uncertainty conditions. We considered four different metrics namely the probability of detection, probability of false alarm, channel utilization rate and the user service rate to analyse each technique. We investigated each technique to understand their respective performances under the noise-only and signal-plus-noise 
conditions. We present our findings in the following subsections.

\subsection{Performance in the Noise Only Condition, $\mathcal{H}_{0}$}

In the noise-only condition, there is an absence of signals that results in the Signal to Noise Ratio $(S N R)$ of $S N R=0 \mathrm{~dB}$. This implies that we analysed only the probability of false alarm since no signals can be detected in this case. In Table 1 , results show that when the spectra data contains only noise samples without any uncertainty (i.e. noise uncertainty $=0 \mathrm{~dB}$ ), then the channel utilisation rate (CU) under the fixed threshold method is $100 \%$. This implies that the entire channel is free and is fully utilized by the users in the network. This case occurred in our experiments because the fixed threshold value was configured at the noise level without noise uncertainty. Nevertheless, this ideal condition rarely occurs in real-life because noise uncertainties do always occur in communication systems. Consequently, we studied the case of noise uncertainties with results provided in Table 1 and Table 2. Observe that the CU and user service rate (USR) of the fixed method drops to $49.74 \%$ and $38.82 \%$ respectively when the noise uncertainty changes from $0 \mathrm{~dB}$ to $1 \mathrm{~dB}$. This drop in the $\mathrm{CU}$ rate of the fixed threshold method from $100 \%$ to $49.74 \%$ indicates that the performance of the fixed threshold method plummets by $50.26 \%$ when the noise uncertainty

Table 1. Performance of different algorithms under the complete noisy environment and the with introduction of certain value of noise uncertainty (all CU and USR values are in percentages and Th means threshold).

\begin{tabular}{|c|c|c|c|c|c|c|c|c|}
\hline \multirow{2}{*}{ Algorithms } & \multicolumn{4}{|c|}{ Noise uncertainty $=0 \mathrm{~dB}$} & \multicolumn{4}{|c|}{ Noise uncertainty $=1 \mathrm{~dB}$} \\
\hline & Th & $\mathcal{P}_{\mathcal{F A}}$ & $\mathrm{CU}$ & USR & Th & $\mathcal{P}_{\mathcal{F A}}$ & $\mathrm{CU}$ & USR \\
\hline Fixed & 1 & 0.0283 & 100 & 40.083 & 1 & 0.042 & 49.748 & 38.82 \\
\hline Modified Otsu & 0.6001 & 0.0461 & 73.979 & 36.9305 & 1.2942 & 0.0212 & 78.036 & 36.966 \\
\hline ROHT & 1.0877 & 0.0224 & 99.606 & 40.1145 & 1.9803 & $8.54 \mathrm{E}-03$ & 99.225 & 40.1025 \\
\hline FOST & 0.7461 & 0.0268 & 98.856 & 40.132 & 1.8865 & 0.0105 & 98.043 & 40.147 \\
\hline FCME & 0.1511 & 0.0592 & 47.422 & 40.05 & 0.915 & 0.0218 & 58.89 & 40.068 \\
\hline
\end{tabular}

Table 2. Performance of different algorithms under the different values of the noise uncertainties (all CU and USR values are in percentages and Th means threshold).

\begin{tabular}{|c|c|c|c|c|c|c|c|c|c|c|c|c|}
\hline \multirow{2}{*}{ Algorithms } & \multicolumn{4}{|c|}{ Noise uncertainty $=2 \mathrm{~dB}$} & \multicolumn{4}{|c|}{ Noise uncertainty $=3 \mathrm{~dB}$} & \multicolumn{4}{|c|}{ Noise uncertainty $=4 \mathrm{~dB}$} \\
\hline & Th & $\mathcal{P}_{\mathcal{F A}}$ & $\mathrm{CU}$ & USR & Th & $\mathcal{P}_{\mathcal{F A}}$ & $\mathrm{CU}$ & USR & Th & $\mathcal{P}_{\mathcal{F A}}$ & $\mathrm{CU}$ & USR \\
\hline Fixed & 1 & 0.043 & 25.033 & 20.0535 & 1 & 0.0427 & 16.664 & 12.185 & 1 & 0.0429 & 12.619 & 8.461 \\
\hline Modified Otsu & 2.19 & $6.94 \mathrm{E}-03$ & 77.092 & 36.975 & 2.9493 & $2.42 \mathrm{E}-03$ & 75.769 & 37 & 3.3832 & $9.21 \mathrm{E}-04$ & 75.043 & 37.049 \\
\hline ROHT & 3.17 & $1.95 \mathrm{E}-03$ & 99.37 & 40.1095 & 4.2244 & $4.78 \mathrm{E}-04$ & 99.484 & 40.0515 & 4.171 & $1.20 \mathrm{E}-04$ & 99.542 & 40.1187 \\
\hline FOST & 3.27 & $2.60 \mathrm{E}-03$ & 98.243 & 40.019 & 3.6497 & $6.85 \mathrm{E}-04$ & 98.691 & 40.16 & 4.1928 & $1.92 \mathrm{E}-04$ & 98.6995 & 40.1027 \\
\hline FCME & 1.23 & 0.0115 & 55.137 & 40.0425 & 2.172 & $7.30 \mathrm{E}-03$ & 51.555 & 39.9715 & 2.0395 & $4.90 \mathrm{E}-03$ & 50.0605 & 40.212 \\
\hline
\end{tabular}


level was increased only from $0 \mathrm{~dB}$ to $1 \mathrm{~dB}$. Notice further that the $\mathrm{CU}$ rate of the fixed threshold method decreased continuously by about $50 \%$ as we increased the noise uncertainty level by $1 \mathrm{~dB}$ (see Table 1 and Table 2). This implies that the fixed threshold method cannot be effective in noise uncertainty regimes. On the other hand, notice that the CU of each DTE increased as the noise uncertainty changed from $0 \mathrm{~dB}$ to $1 \mathrm{~dB}$. By averaging these increments across the four different DTEs, we observed an approximate average increase of $4 \%$ in the CU rate. This occurred because the DTE techniques increased their respective threshold values according to the increase in the noise level, thus guaranteeing more free bands to be used by the CR users. The ROHT and FOST techniques experienced very high bandwidth utilization values compared to the fixed threshold technique in the no noise uncertainty condition. The Modified Otsu algorithm adjusted its threshold value automatically to a level, which resulted in the low bandwidth channel utilisation compared to the fixed, ROHT and the FOST techniques. The lowest bandwidth utilisation was experienced by the FCME technique. For the USR, the auction model ensured that the highest number of users was served for the fixed, ROHT, FOST and the FCME techniques while the lowest USR was experienced for the Modified Otsu technique. This implies that users will benefit more from using DTEs such as the ROHT, FOST, FCME over the fixed threshold method. We also considered the probability of the false alarm, which was lowest for the ROHT and FOST methods. This implies that these methods can be trusted in applications that require maximum spectra utilization at the expense of PU interference.

We considered conditions with increasing noise uncertainty rates and present our results in Table 2. As expected, the CU reduces drastically for the fixed threshold technique but remains almost constant for the ROHT, FOST and the Modified Otsu technique. For the FCME, the CU increased by approximately $10 \%$. Analysing the USR, it remains almost the same for the ROHT, FCME, FOST and for the Modified Otsu methods. However, the USR decreased by $4 \%$ for the fixed threshold technique. The lowest $\mathcal{P}_{\mathcal{F A}}$ is experienced by the ROHT. Thus we concluded that as the noise uncertainty increases the performance of the fixed threshold technique reduces drastically. However, the ROHT, FOST, FCME and the Modified Otsu techniques maintain their overall performance.

Furthermore, we show in Table 2 that the performance of the fixed threshold method decreases steadily with increase in noise uncertainty, which indicates that the fixed threshold method is not ideal for the energy detector even under the auction game model. Thus, DTE techniques like the ROHT, FOST, FCME and the Modified Otsu algorithm play a very significant role in improving the overall performance of the energy detector. It is indicated in Table 1 and Table 2 that the CU and USR for the DTE techniques are maintained under a constant increase in the noise uncertainty level. Among the DTE techniques that we investigated, the performance of the ROHT stands out irrespective of the increase in the noise uncertainties. This makes it a better performer in noise-only conditions. 


\subsection{Performance in the Signal plus Noise Condition, $\mathcal{H}_{1}$}

In the signal plus noise condition, the interference level to the PU becomes an important parameter. From Table 3, it is seen that the fixed threshold technique performs least based on its channel utilisation (CU) rate. It utilises only $20 \%$ of the entire channel. In addition, only $10 \%$ of the users are served using this technique via the application of the auction game model. These results imply that the fixed threshold method may remain undependable even in the signal plus noise conditions and we advise against its use in the ED. The ROHT provides the best performance based on its USR of $30 \%$ as analysed from Table 3 .

The FOST and the Modified Otsu technique performed similarly in terms of their $\mathrm{CU}$ rates. Both techniques utilised the channel more efficiently, however, their USR falls below 50\%. The FCME technique failed to utilise maximally the channel based on its low $\mathrm{CU}$ and the USR. Considering the probabilities of false alarm, the ROHT had the least and the fixed technique had the highest probability. As we increased the noise uncertainty as shown in Table 4, only the performance of the fixed threshold technique deteriorated while other techniques maintained good CU and USR rates. However, except the FCME technique, the probability of false alarm increased for all other techniques. Thus for the present case, we can conclude that the ROHT provides the best performance for the auction game model based one tier CRN.

Table 3. Performance of different algorithms under the different values of the noise uncertainties at a fixed $S N R=10 \mathrm{~dB}$ (all CU and USR values are in percentages and Th means threshold).

\begin{tabular}{ccccccccccc}
\hline \multirow{2}{*}{ Algorithms } & \multicolumn{4}{c}{ Noise uncertainty $=\mathbf{0 ~ d B}$} & \multicolumn{5}{c}{ Noise uncertainty = 1 dB } \\
\cline { 2 - 10 } & Th & $\mathcal{P}_{\mathcal{D}}$ & $\mathcal{P}_{\mathcal{F A}}$ & CU & USR & Th & $\mathcal{P}_{\mathcal{D}}$ & $\mathcal{P}_{\mathcal{F A}}$ & CU & USR \\
\hline Fixed & 1 & 1 & 0.0786 & 20 & 10 & 1 & 1 & 0.0957 & 10 & 5 \\
Modified Otsu & 5.3564 & 1 & $1.79 \mathrm{E}-14$ & 90 & 40 & 6.3564 & 1 & $8.37 \mathrm{E}-12$ & 90 & 40 \\
ROHT & 6.6112 & 1 & $4.40 \mathrm{E}-21$ & 100 & 30 & 7.6112 & 0.996 & $1.16 \mathrm{E}-15$ & 100 & 35 \\
FOST & 6.2233 & 1 & $6.78 \mathrm{E}-19$ & 90 & 30 & 7.2233 & 0.9993 & $1.34 \mathrm{E}-14$ & 90 & 35 \\
FCME & 2.2 & 1 & $9.31 \mathrm{E}-04$ & 50 & 25 & 3.2 & 1 & $1.15 \mathrm{E}-04$ & 50 & 20 \\
\hline
\end{tabular}

Table 4. Performance of different algorithms under no noise uncertainty and at different values of SNR (all CU and USR values are in percentages and Th means threshold).

\begin{tabular}{|c|c|c|c|c|c|c|c|c|c|c|c|c|c|c|c|}
\hline \multirow{2}{*}{ Algorithms } & \multicolumn{5}{|c|}{$S N R=3 \mathrm{~dB}$} & \multicolumn{5}{|c|}{$S N R=5 \mathrm{~dB}$} & \multicolumn{5}{|c|}{$S N R=10 \mathrm{~dB}$} \\
\hline & Th & $\mathcal{P}_{\mathcal{D}}$ & $\mathcal{P}_{\mathcal{F A}}$ & $\mathrm{CU}$ & USR & Th & $\mathcal{P}_{\mathcal{D}}$ & $\mathcal{P}_{\mathcal{F A}}$ & $\mathrm{CU}$ & USR & $\mathrm{Th}$ & $\mathcal{P}_{\mathcal{D}}$ & $\mathcal{P}_{\mathcal{F A}}$ & $\mathrm{CU}$ & USR \\
\hline Fixed & 1 & 0.99 & 0.0786 & 40 & 18.5 & 1 & 1 & 0.0786 & 60 & 30 & 1 & 1 & 0.0786 & 20 & 10 \\
\hline Modified Otsu & 3.23 & 0.37 & $2.46 \mathrm{E}-06$ & 70 & 30.5 & 2.71 & 0.9994 & $6.15 \mathrm{E}-05$ & 80 & 35 & 5.36 & 1 & $1.79 \mathrm{E}-14$ & 90 & 40 \\
\hline ROHT & 4.7 & $8.33 \mathrm{E}-03$ & $1.61 \mathrm{E}-11$ & 100 & 42.5 & 3.73 & 0.9643 & $6.89 \mathrm{E}-08$ & 100 & 35 & 6.61 & 1 & $4.40 \mathrm{E}-21$ & 100 & 30 \\
\hline FOST & 4.4 & 0.02 & $2.45 \mathrm{E}-10$ & 100 & 40.5 & 3.48 & 0.984 & $4.17 \mathrm{E}-07$ & 100 & 45 & 6.22 & 1 & $6.78 \mathrm{E}-19$ & 90 & 30 \\
\hline FCME & 0.55 & 0.99 & 0.2195 & 30 & 14 & 0.326 & 1 & 0.3231 & 30 & 15 & 2.2 & 1 & $9.31 \mathrm{E}-04$ & 50 & 25 \\
\hline
\end{tabular}


Table 4 provides the performance of different algorithms using different $S N R$ values. Beginning with the low $S N R$ values, that is, at $S N R=3 \mathrm{~dB}$, both the ROHT and the FOST techniques performed well based on their $\mathrm{CU}$ rates and USR, which was greater than $40 \%$. The Modified Otsu algorithm performed comparably to the ROHT and the FOST, however, the FCME technique performed least providing low CU and USR rates. The FCME algorithm may have performed poorly because of its highly sensitive parameter values, whose values may not have been suitable for this use-case. Since we used the same FCME parameter values in the noise only case for the signal plus noise case, we note that this may have been a drawback for the FCME algorithm. Consequently, users may always need to change the FCME parameters whenever the sensing conditions change, which is not realistic, thus limiting the FCME's performance. Thus, there is need to adapt automatically the parameters of the FCME algorithm and perhaps other DTEs too. By increasing the $S N R$, the ROHT and the FOST techniques maintained their performance levels in terms of their CUs albeit with an irregular USR behaviour (as in Table 4), which increased and decreased abnormally. At the time of our experiments, we could only attribute this irregularity of the ROHT and FOST methods to the increasing and decreasing variance in the dataset, noting that the variance is a major variable used by both methods to compute threshold values. The Modified Otsu algorithm performed well in terms of its CU and USR even as the $S N R$ values were increased. The FCME algorithm also performed well until the $S N R=5 \mathrm{~dB}$ was reached. Based on the results obtained, the ROHT technique provided the lowest probability of false alarm considering all $S N R$ values thus been the best performer.

\section{Conclusion}

In this paper, we have analysed the performance of some dynamic threshold estimation (DTE) techniques under both noise-only and signal-plus-noise conditions using the auction game model based on the one-tier CRN. We reveal that when the auction game model is used to optimize the one tier CRN, the ROHT technique provides the best DTE performance in terms of its high channel utilization (CU), user service rate (USR) and its low probability of false alarm. Our analysis showed that the fixed threshold technique performed well only under the zero noise-uncertainty level, which usually does not exist in real-life situations. In future works, we will extend our study to investigate more DTE techniques using the auction game model for the two tier CRNs.

\section{Conflicts of Interest}

The authors declare no conflicts of interest regarding the publication of this paper.

\section{References}

[1] Haykin, S. (2005) Cognitive Radio: Brain-Empowered Wireless Communications. 
IEEE Journal on Selected Areas in Communications, 23, 201-220. https://doi.org/10.1109/JSAC.2004.839380

[2] Kolodzy, P. (2001) Next Generation Communications: Kickoff Meeting. Vol. 10, Proceedings of Defense Advanced Research Projects Agency, Arlington, 17 October 2001, 199-201.

[3] Mitola, J. (1999) Cognitive Radio for Flexible Mobile Multimedia Communications. IEEE International Workshop on Mobile Multimedia Communications, San Diego, 15-17 November 1999, 3-10. https://doi.org/10.1109/MOMUC.1999.819467

[4] Goldsmith, A., Jafar, S.A., Maric, I. and Srinivasa, S. (2009) Breaking Spectrum Gridlock with Cognitive Radios: An Information Theoretic Perspective. Proceedings of the IEEE, 97, 894-914. https://doi.org/10.1109/JPROC.2009.2015717

[5] Akyildiz, I.F., Lee, W.Y., Vuran, M.C. and Mohanty, S. (2006) NeXt Generation/Dynamic Spectrum Access/Cognitive Radio Wireless Networks: A Survey. Computer Networks, 50, 2127-2159. https://doi.org/10.1016/j.comnet.2006.05.001

[6] Wang, B. and Liu, K.R. (2011) Advances in Cognitive Radio Networks: A Survey. IEEE Journal of Selected Topics in Signal Processing, 5, 5-23.

https://doi.org/10.1109/JSTSP.2010.2093210

[7] Yucek, T. and Arslan, H. (2009) A survey of Spectrum Sensing Algorithms for Cognitive Radio Applications. IEEE Communications Surveys \& Tutorials, 11, 116-130. https://doi.org/10.1109/SURV.2009.090109

[8] Akyildiz, I.F., Lee, W.Y. and Chowdhury, K.R. (2009) Spectrum Management in Cognitive Radio Ad Hoc Networks. IEEE Network, 23, 6-12.

https://doi.org/10.1109/MNET.2009.5191140

[9] Ramani, V. and Sharma, S.K. (2017) Cognitive Radios: A Survey on Spectrum Sensing, Security and Spectrum Handoff. China Communications, 14, 185-208. https://doi.org/10.1109/CC.2017.8233660

[10] Akyildiz, I.F., Lee, W.Y. and Chowdhury, K.R. (2009) CRAHNs: Cognitive Radio ad hoc Networks. Ad Hoc Networks, 7, 810-836. https://doi.org/10.1016/j.adhoc.2009.01.001

[11] Cabric, D., Mishra, S.M. and Brodersen, R.W. (2004) Implementation Issues in Spectrum Sensing for Cognitive Radios. IEEE Conference Record of the Thirty-Eighth Asilomar Conference on Signals, Systems and Computers, Pacific Grove, 7-10 November 2004, 772-776. https://doi.org/10.1109/ACSSC.2004.1399240

[12] Wu, J., Luo, T. and Yue, G. (2009, December) An Energy Detection Algorithm Based on Double-Threshold in Cognitive Radio Systems. IEEE 2009 First International Conference on Information Science and Engineering, Nanjing, 26-28 December 2009, 493-496. https://doi.org/10.1109/ICISE.2009.257

[13] Xie, J. and Chen, J. (2012) An Adaptive Double-Threshold Spectrum Sensing Algorithm under Noise Uncertainty. 2012 IEEE 12th International Conference on Computer and Information Technology (CIT), Chengdu, 27-29 October 2012, 824-827. https://doi.org/10.1109/CIT.2012.171

[14] Liu, S.Q., Hu, B.J. and Wang, X.Y. (2012) Hierarchical Cooperative Spectrum Sensing Based on Double Thresholds Energy Detection. IEEE Communications Letters, 16, 1096-1099. https://doi.org/10.1109/LCOMM.2012.050112.120765

[15] Onumanyi, A.J., Abu-Mahfouz, A.M. and Hancke, G.P. (2018) A Comparative Analysis of Local and Global Adaptive Threshold Estimation Techniques for Energy Detection in Cognitive Radio. Physical Communication, 29, 1-11. https://doi.org/10.1016/j.phycom.2018.04.008

[16] Onumanyi, A.J., Onwuka, E.N., Aibinu, A.M., Ugweje, O.C. and Salami, M.J.E. 
(2014) A Real Valued Neural Network Based Autoregressive Energy Detector for Cognitive Radio Application. International Scholarly Research Notices, 2014, Article ID: 579125. https://doi.org/10.1155/2014/579125

[17] Bagwari, A. and Tomar, G.S. (2013) Two-Stage Detectors with Multiple Energy Detectors and Adaptive Double Threshold in Cognitive Radio Networks. International Journal of Distributed Sensor Networks, 9, Article ID: 656495. https://doi.org/10.1155/2013/656495

[18] Avila, J. and Thenmozhi, K. (2015) Adaptive Double Threshold with Multiple Energy Detection Technique in Cognitive Radio. Research Journal of Applied Sciences, Engineering and Technology, 10, 1336-1342. https://doi.org/10.19026/rjaset.10.1831

[19] Ahuja, B., Sharma, K., Abo-Zahhad, M., Amin, O., Farrag, M., Ali, A. and Rachman, A. (2014) Adaptive Double Threshold Based Spectrum Sensing for Cognitive Radio Networks. International Journal of Energy, Information and Communications, 5, 1-16. https://doi.org/10.14257/ijeic.2014.5.6.01

[20] Dubey, R.K. and Verma, G. (2015) Improved Spectrum Sensing for Cognitive Radio Based on Adaptive Double Threshold. International Journal of Emerging Trends in Electrical and Electronics, 11, 1-6.

[21] Ling, X., Wu, B., Wen, H., Ho, P.H., Bao, Z. and Pan, L. (2012) Adaptive Threshold Control for Energy Detection Based Spectrum Sensing in Cognitive Radios. IEEE Wireless Communications Letters, 1, 448-451. https://doi.org/10.1109/WCL.2012.062512.120299

[22] Wang, N., Gao, Y. and Cuthbert, L. (2014) Spectrum Sensing Using Adaptive Threshold Based Energy Detection for OFDM Signals. IEEE International Conference on Communication Systems, Macau, 19-21 November 2014, 359-363. https://doi.org/10.1109/ICCS.2014.7024825

[23] Lehtomaki, J.J., Vartiainen, J., Juntti, M. and Saarnisaari, H. (2006) Spectrum Sensing with Forward Methods. Military Communications Conference, Washington DC, 23-25 October 2006, 1-7.

[24] Lehtomaki, J.J., Juntti, M. and Saarnisaari, H. (2005) CFAR Strategies for Channelized Radiometer. IEEE Signal Processing Letters, 12, 13-16.

https://doi.org/10.1109/LSP.2004.839701

[25] Lehtomaki, J.J., Vuohtoniemi, R. and Umebayashi, K. (2013) On the Measurement of Duty Cycle and Channel Occupancy Rate. IEEE Journal on Selected Areas in Communications, 31, 2555-2565. https://doi.org/10.1109/JSAC.2013.131114

[26] Lehtomaki, J.J., Vartiainen, J., Juntti, M. and Saarnisaari, H. (2007) CFAR Outlier Detection with Forward Methods. IEEE Transactions on Signal Processing, 55, 4702-4706. https://doi.org/10.1109/TSP.2007.896239

[27] Onumanyi, A.J., Onwuka, E.N., Aibinu, A.M., Ugweje, O. and Salami, M.J.E. (2014) Effect of Spectrum Occupancy on the Performance of a Real Valued Neural Network Based Energy Detector. International Joint Conference on Neural Networks, Beijing, 6-11 July 2014, 1191-1196.

[28] Ohize, H., Onumanyi, A.J., Dlodlo, M. and Bello-Salau, H. (2017) An Adaptive Wavelet-Based Scale Space Filtering Algorithm for Spectrum Sensing in Cognitive Radio. Wireless Communications and Networking Conference, San Francisco, 19-22 March 2017, 1-6. https://doi.org/10.1109/WCNC.2017.7925633

[29] Joshi, D.R., Popescu, D.C. and Dobre, O.A. (2010) Adaptive Spectrum Sensing with Noise Variance Estimation for Dynamic Cognitive Radio Systems. 44th Annual Conference on Information Sciences and Systems, Princeton, 17-19 March 2010, 1-5. https://doi.org/10.1109/CISS.2010.5464913 
[30] Srivastava, S.K. and Banerjee, A. (2009) "n-Ratio" Logic Based Cooperative Spectrum Sensing Using Double Threshold Energy Detection. Energy, 3, 4.

[31] Teo, D., Zhong, K. and Ng, B.C. (2010) An Iterative Threshold Selection Algorithm for Cooperative Sensing in a Cognitive Radio Network. IEEE Symposium on New Frontiers in Dynamic Spectrum, Singapore, 6-9 April 2010, 1-8. https://doi.org/10.1109/DYSPAN.2010.5457843

[32] Datla, D., Wyglinski, A.M. and Minden, G.J. (2005) A Statistical Approach to Spectrum Measurement Processing. Information and Telecommunication Technology Center.

[33] Datla, D., Wyglinski, A.M. and Minden, G.J. (2009) A Spectrum Surveying Framework for Dynamic Spectrum Access Networks. IEEE Transactions on Vehicular Technology, 58, 4158-4168. https://doi.org/10.1109/TVT.2009.2021601

[34] Gorcin, A., Qaraqe, K.A., Celebi, H. and Arslan, H. (2010) An Adaptive Threshold Method for Spectrum Sensing in Multi-Channel Cognitive Radio Networks. IEEE 17 th International Conference on Telecommunications, Doha, 4-7 April 2010, 425-429. https://doi.org/10.1109/ICTEL.2010.5478783

[35] Onumanyi, A.J., Onwuka, E.N., Aibinu, A.M., Ugweje, O.C. and Salami, M.J.E. (2017) A Modified Otsu's Algorithm for Improving the Performance of the Energy Detector in Cognitive Radio. International Journal of Electronics and Communications, 79, 53-63. https://doi.org/10.1016/j.aeue.2017.04.013

[36] Barnes, S.D., Van Vuuren, P.J. and Maharaj, B.T. (2013) Spectrum Occupancy Investigation: Measurements in South Africa. Measurement, 46, 3098-3112. https://doi.org/10.1016/j.measurement.2013.06.010

[37] Rajasekharan, J., Eriksson, J. and Koivunen, V. (2011) Cooperative Game Theory and Auctioning for Spectrum Allocation in Cognitive Radios. IEEE 22nd International Symposium on Personal Indoor and Mobile Radio Communications, 656-660. https://doi.org/10.1109/PIMRC.2011.6140044

[38] Stanojev, I., Simeone, O., Spagnolini, U., Bar-Ness, Y. and Pickholtz, R.L. (2010) Cooperative ARQ via Auction-Based Spectrum Leasing. IEEE Transactions on Communications, 58, 1843-1856. https://doi.org/10.1109/TCOMM.2010.06.080575

[39] Chakraborty, T., Misra, I.S. and Manna, T. (2016) Design and Implementation of VoIP Based Two-Tier Cognitive Radio Network for Improved Spectrum Utilization. IEEE Systems Journal, 10, 370-381. https://doi.org/10.1109/JSYST.2014.2382607

[40] Roy, A., Midya, S., Majumder, K., Phadikar, S. and Dasgupta, A. (2017) Optimized Secondary User Selection for Quality of Service Enhancement of Two-Tier Multi-User Cognitive Radio Network: A Game Theoretic Approach. Computer Networks, 123, 1-18. https://doi.org/10.1016/j.comnet.2017.05.002 